Original Paper $\quad$ http://ajol.info/index.php/ijbcs $\quad$ http://indexmedicus.afro.who.int

\title{
Caractérisation physico-chimique des eaux d'un système lacustre du cordon littoral ivoirien : cas du lac Labion
}

\author{
Ella ODJOHOU ${ }^{1 *}$, Mireille AMANI ${ }^{1}$, Gbombélé SORO ${ }^{2}$ et Sylvain MONDE ${ }^{1}$ \\ ${ }^{1}$ Laboratoire des Géosciences marines, UFR SRTM, Université Félix Houphouët Boigny, 22 B.P. 582 Abidjan \\ 22, Côte d'Ivoire. \\ ${ }^{2}$ Laboratoire des sciences de l'eau, UFR STRM, Université Félix Houphouët Boigny, 22 B.P. 582 Abidjan 22, \\ Côte d'Ivoire. \\ *Auteur correspondant ; E-mail : ellaodjohou@gmail.com
}

\section{RESUME}

L'analyse spatio-temporelle des paramètres physico-chimiques des eaux du lac Labion dans le système lagunaire ivoirien, a été abordé dans un contexte de variabilité climatique. L'objectif est de caractériser la physico-chimie des eaux au cours des saisons climatiques. Pour y parvenir, une analyse hydrologique a été réalisé à partir de mesure in situ des paramètres $\mathrm{pH}$, Température, Conductivité électrique, Oxygène dissous, Matières en suspension, Transparence et Turbidité. Ces paramètres ont été mesurés à l'aide d'un multi-paramètre. Celuici est mis à l'eau et les résultats s'affichent à l'écran. Seule la transparence a été mesuré à l'aide du disque de Secchi. La température $\left(31^{\circ} \mathrm{C}\right)$ indique des eaux relativement chaudes. Quant au $\mathrm{pH}$, il varie du neutre $(6,79)$ à alcalin $(8,99)$. La conductivité moyenne sur le lac est de $40 \mu \mathrm{S} / \mathrm{cm}$. Les eaux sont bien oxygénées $(7$ à $8 \mathrm{mg} / \mathrm{L})$ et présente peu de matières en suspension. L'analyse en composante principale montre une forte corrélation $(\mathrm{r}>$ $0,7)$ entre les variables température, oxygène, $\mathrm{pH}$ et conductivité. Ces résultats mettent en évidence deux $(02)$ mécanismes qui sont à l'origine des forçages naturel et artificiel. Il s'agit du mécanisme de la photosynthèse occasionnant une bonne oxygénation des eaux et du mécanisme de pollution traduisant une forte turbidité.

(C) 2020 International Formulae Group. All rights reserved.

Mots clés : Lac, saison climatique, paramètre, mécanisme, évolution.

\section{Physico-chemical characterization of the waters of a lake system of the ivorian coastal cordon: case of Lake Labion}

\begin{abstract}
The spatio-temporal analysis of the physicochemical parameters of the waters of Lake Labion in the Ivorian lagoon system was tackled in the context of climate variability. The objective is to characterize the physico-chemistry of the waters during the climatic seasons. To achieve this, a hydrological analysis was carried out using in situ measurement of the parameters $\mathrm{pH}$, Temperature, Electrical conductivity, Dissolved oxygen, Suspended matter, Transparency and Turbidity. These parameters were measured using a multi-parameter. It is launched and the results are displayed on the screen. Only transparency was measured using the Secchi disk. The temperature $\left(31^{\circ} \mathrm{C}\right)$ indicates relatively warm waters. As for the $\mathrm{pH}$, it varies from neutral (6.79) to alkaline (8.99). The average conductivity on the lake is $40 \mu \mathrm{S} / \mathrm{cm}$. The waters are well oxygenated (7 to $8 \mathrm{mg} / \mathrm{L})$ and
\end{abstract}


have little suspended matter. The principal component analysis shows a strong correlation $(r>0.7)$ between the variables temperature, oxygen, $\mathrm{pH}$ and conductivity. These results highlight two (02) mechanisms which are at the origin of natural and artificial forcings. This is the mechanism of photosynthesis causing good oxygenation of water and the pollution mechanism reflecting high turbidity.

(C) 2020 International Formulae Group. All rights reserved.

Keywords: Lake, climatic season, parameter, mechanism, evolution.

\section{INTRODUCTION}

La Côte d'Ivoire possède le plus grand système lagunaire de l'Afrique de l'Ouest. Ce système se compose des lagunes de GrandLahou, Aby et Ebrié. La lagune Ebrié, sur laquelle repose une diversité de milieux naturels (lac, rivière, cours d'eau, etc.), présente une superficie de $532 \mathrm{Km}^{2}$. En effet, les lacs sont des dépressions naturelles remplis d'eau et sans connexion avec l'océan. Ils présentent des caractéristiques surfaciques permettant une stratification de eaux (Touchart, 2000; Aka, 2016). Les lacs, qui sont des milieux confinés, constituent des réserves en eau susceptibles d'approvisionner des populations. Ces environnements lacustres connaissent des problèmes de pollution dus aux forçages (naturel et anthropique) qui pourraient impacter la qualité de l'eau et par la suite à l'état de santé influençant ainsi la mortalité à la fois chez l'homme et les animaux (Kazi et al., 2009). Cette étude vise donc à caractériser les paramètres physico-chimiques des eaux du lac au cours des saisons climatiques puis de ressortir le degré de corrélation existant entre les variables étudiées.

\section{Présentation de la zone d'étude}

Le lac Labion, situé au Sud de la Côte d'Ivoire, est compris entre la longitude $5^{\circ} 13^{\prime} 0^{\prime \prime}$ ' et $5^{\circ} 15^{\prime} 0^{\prime \prime}$ Nord et la latitude $4^{\circ} 12^{\prime} 0^{\prime}$ " et $4^{\circ} 8^{\prime} 0^{\prime \prime}$ ' Ouest. Sa superficie est de 480,20 hectares.

\section{Station d'échantillonnage}

L'échantillonnage des paramètres physico-chimiques s'est réalisée sur onze (11) stations au cours des quatre (04) saisons climatiques dont 02 saisons sèches (Janvier et Septembre 2017) et 02 saisons pluvieuses (juin et novembre 2017). Ces échantillonnages ont été fait en surface puis dans la colonne d'eau sur chaque station.

\section{MATERIEL ET METHODES \\ Matériel}

Le matériel utilisé se compose essentiellement d'un multi-paramètre, un GPS et un disque de Secchi. Composé d'une sonde et d'un écran, le multi-paramètre a servi à la mesure in situ des paramètres physicochimiques ( $\mathrm{pH}$, Température, Oxygène dissous, Conductivité, Matières en suspension, Turbidité). Le GPS a été utilisé pour localiser les stations d'échantillonnage. Quant au disque de Secchi, il a permis la mesure de la transparence.

\section{Méthodes \\ Acquisition des données physico-chimiques}

Les mesures in situ des paramètres ont été faites immédiatement dans le lac à l'aide d'un multi-paramètre. Pour la mesure de ces paramètres (pH, Température, Oxygène dissous, Conductivité, Turbidité), on procède au calibrage du multi-paramètre depuis l'embarcation puis à sa mise dans l'eau à chaque station d'échantillonnage. Les valeurs obtenues s'affichent à l'écran où elles sont soigneusement relevées par l'opérateur. Pour la mesure de la transparence, le principe consiste à descendre le disque de Secchi dans l'eau à l'aide d'une corde graduée jusqu'à sa disparition. La hauteur d'eau à partir de laquelle le disque n'est plus visible par l'opérateur est noté en mètre (m).

\section{Elaboration des cartes de répartition}

La réalisation des cartes de répartition spatiale a été élaboré avec le logiciel ArcGis. Celui-ci se fait en deux (02) dont la première 
consiste à définir un tableur Excel en fichier CVS. La carte de contour obtenue par digitalisation dans la base de données du système lagunaire Ebrié, est ajouté au fichier CVS. La deuxième étape permet de ressortir les cartes de répartition spatiale des différents paramètres en choisissant le Krigeage comme méthode adéquate d'interpolation.

\section{Corrélation des paramètres physico- chimiques par analyse en composante principale}

Les données collectées lors de la campagne d'échantillonnage ont été soumises à des analyses statistique avec le logiciel Statistica 7.1 pour une meilleure interprétation des résultats. L'analyse en composante principale (ACP) qui est un outil exploratoire, consiste à chercher des combinaisons linéaires des variables quantitatives en vue de former des axes factoriels afin de détecter les individus ressemblants, ensuite de mettre en évidence une typologie d'individus et enfin de détecter les relations entre les différentes variables. L'ACP se fera sur onze (11) unité statistiques pour six (06) variables $(\mathrm{pH}$, Température, Oxygène dissous, Conductivité électrique, Turbidité et Transparence).

\section{RESULTATS}

\section{Répartition spatio-temporelle du potentiel d'hydrogène \\ Saison sèche (janvier et septembre)}

La répartition spatiale du $\mathrm{pH}$ sur le plan d'eau du lac n'est pas homogène pendant la grande et la petite saison sèche. En effet, durant la grande saison sèche, le $\mathrm{pH}$ varie de 6,7 à 9,7 (Figure 3) tandis qu'au cours de la petite saison sèche, il part de 6,88 à 8,36 (Figure 4). L'évolution en surface du $\mathrm{pH}$ met en évidence des zones (Centre) basiques (pH 7) pendant la grande saison sèche qui deviennent acides au cours de la petite saison sèche.

\section{Saison pluvieuse (juillet et novembre)}

Pendant la grande saison pluvieuse, les eaux du lac Labion sont quasi-acides avec des valeurs de $\mathrm{pH}$ en majorité inférieure à 7 (Figure 5) tandis qu'au cours de la petite saison pluvieuse (Figure 6), il varie du neutre $(7,84)$ à alcalin $(9,15)$. Toutefois, il présente une répartition spatiale hétérogène sur les deux (02) saisons (Figure 4). Le Sud-ouest du lac présente un $\mathrm{pH}$ basique $(8,09)$ pendant la grande saison pluvieuse. Cette même zone présente au cours de la petite saison pluvieuse, un $\mathrm{pH}$ quasiment neutre $(7,84)$.

\section{Evolution spatio-temporelle de la température}

\section{Saison sèche (janvier et septembre)}

La température au cours de la saison sèche est quasiment constante en surface car elle varie peu (Figure 7). Elle part de $30,3{ }^{\circ} \mathrm{C}$ à $32^{\circ} \mathrm{C}$ pendant la grande saison et de 29,1 à 30,6 ${ }^{\circ} \mathrm{C}$ pendant la petite saison sèche. Dans la colonne d'eau, l'évolution de la température connait une légère variation pendant la petite saison sèche. Les valeurs chutent et atteignent un minimal de $28{ }^{\circ} \mathrm{C}$ en profondeur.

\section{Saison pluvieuse (juillet et novembre)}

Les températures enregistrées pendant la grande saison pluvieuse, varient de $26,7{ }^{\circ} \mathrm{C}$ à $29,1{ }^{\circ} \mathrm{C}$ en surface. Dans la colonne d'eau, celles-ci et atteignent un minimale de $25^{\circ} \mathrm{C}$. en revanche, la station 10 reste constante sur toute la colonne d'eau. Au cours de la petite saison pluvieuse, les valeurs de la température remontent légèrement en surface et varient de $31{ }^{\circ} \mathrm{C}$ à $21,9^{\circ} \mathrm{C}$. la température varie peu dans la colonne d'eau pendant la cette saison (Figure 8).

\section{Evolution de l'oxygène dissous \\ Saison sèche (janvier et septembre)}

Les teneurs en oxygène dissous à la surface du lac pendant la grande saison sèche varient peu $(8,2 \mathrm{mg} / \mathrm{l}$ à $8,85 \mathrm{mg} / \mathrm{L})$ avec une moyenne de $8,5 \mathrm{mg} / \mathrm{L}$. Quant à la petite saison sèche, elles varient de $8,33 \mathrm{mg} / \mathrm{L}$ à $9,01 \mathrm{mg} / \mathrm{L}$. Dans la colonne d'eau, ces différentes valeurs connaissent une chute et atteignent une valeur minimale de $4,5 \mathrm{mg} / \mathrm{L}$ pendant la grande saison sèche (Figure 9).

\section{Saison pluvieuse (juillet et novembre)}

En saison pluvieuse, l'oxygène dissous varie de $6,14 \mathrm{mg} / \mathrm{L}$ à $8,27 \mathrm{mg} / \mathrm{L}$ au cours de la grande saison et de $8,5 \mathrm{mg} / \mathrm{l}$ à $8,7 \mathrm{mg} / \mathrm{L}$ pendant la petite saison (Figure 10). L'oxygène dissous varie très peu en surface pendant la petite 
saison pluvieuse. Dans la colonne d'eau, l'oxygène reste constant sur les premiers mètres du lac puis connait une baisse dans le fond.

\section{Evolution de la conductivité électrique Saison sèche (janvier et septembre)}

La conductivité électrique pendant la grande saison sèche présente une inégale répartition sur la surface du lac (Figure 11). Elle varie de $40,55 \mu \mathrm{S} / \mathrm{cm}$ à $42,3 \mu \mathrm{S} / \mathrm{cm}$ avec une moyenne de $41,44 \mu \mathrm{S} / \mathrm{cm}$ et un écart-type de 0,64. La partie Est du lac enregistre les valeurs les plus élevées pendant les deux (02) saisons étudiées (Figure 12).

\section{Saison pluvieuse (juillet et novembre)}

La conductivité électrique pendant la saison pluvieuse varie peu et présente une inégale répartition spatiale sur l'ensemble du lac. Au cours de la grande saison pluvieuse, la conductivité varie de $40 \mu \mathrm{S} / \mathrm{cm}$ à $45 \mu \mathrm{S} / \mathrm{cm}$ avec une moyenne de $43,12 \mu \mathrm{S} / \mathrm{cm}$ et un écarttype de 1,69 (Figure 13). Durant la petite saison pluvieuse, on constate une légère baisse de ces valeurs. Celles-ci varient de $40,55 \mu \mathrm{S} / \mathrm{cm}$ à $41,65 \mu \mathrm{S} / \mathrm{cm}$ avec une moyenne de 41,25 $\mu \mathrm{S} / \mathrm{cm}$ et un écart-type de 0,28 . Les fortes conductivités s'observent à la fois dans les parties Ouest et Est du lac pendant la grange saison pluvieuse et dans la partie Est pendant la petite saison pluvieuse (Figure 14).

\section{Evolution saisonnière de la Transparence et de la Turbidité}

Les paramètres transparence et turbidité présentent à l'origine une corrélation inverse. Une turbidité élevée indique une transparence faible. Par définition, la turbidité représente l'ensemble des matières dissoutes minérales et organique. Pour cette étude, seules les cartes de turbidités seront présentées.

\section{Saison sèche (janvier et septembre)}

La turbidité du lac présente une inégale répartition spatiale au cours des deux (02) saisons sèches. Les concentrations en turbidité varient de 4,72 NTU à 6,12 NTU avec une moyenne de 5,47 NTU pendant la grande saison sèche (Figure 15) et de 3,73 NTU à 10,5 NTU avec une moyenne de 5,65 NTU pendant la petite saison sèche (Figure 16). Les fortes valeurs de turbidité se localisent au Centre du lac pendant les deux (02) saisons.

\section{Saison pluvieuse (juillet et novembre)}

La turbidité pendant les saisons, présente une répartition spatiale quasi uniforme. Les turbidités varient de 4 NTU à 10,5 NTU au cours de la grande saison pluvieuse (Figure 17) et de 4 NTU à 7,29 NTU pendant la petite saison pluvieuse (Figure 18). Les faibles turbidités occupent la quasi-totalité du lac.

\section{Corrélation entre les variables étudiées Description statistique des paramètres}

Le tableau 1 présente la description des paramètres statistiques (moyenne, le minimum, le maximum et l'écart-type) des différentes variables étudiés $\left(\mathrm{pH}, \mathrm{T}^{\circ}, \mathrm{CE}, \mathrm{OD}\right.$, Turbidité et Transparence). Le pH varie de l'acidité $(6,79)$ à l'alcalinité $(8,99)$. Quant à la température, les eaux sont relativement chaudes $\left(30,3{ }^{\circ} \mathrm{C}-32\right.$ $\left.{ }^{\circ} \mathrm{C}\right)$. L'oxygène dissous et la conductivité électrique varient très peu. L'oxygène varie de $8,18 \mathrm{mg} / \mathrm{L}$ à $8,76 \mathrm{mg} / \mathrm{L}$ et la conductivité de $40,5 \mu \mathrm{S} / \mathrm{cm}$ à $42,2 \mu \mathrm{S} / \mathrm{cm}$. La transparence de l'eau varie de $0,5 \mathrm{~m}$ à $1,05 \mathrm{~m}$ de profondeur.

\section{Matrice de corrélation}

Le Tableau 2 présente la matrice de corrélation des variables du lac Labion.

L'analyse de la matrice de corrélation ci-dessus présente les corrélations suivantes :

- la température corrèle positivement avec les variables conductivité électrique $(0,77)$ et $\mathrm{pH}$ $(0,70)$;

- l'oxygène dissous (OD) corrèle positivement avec la conductivité électrique d'une façon très significative, avec un coefficient de corrélation de 0,83 .

L'existence d'une corrélation importante entre la température et la conductivité électrique montre qu'il existe une grande affinité chimique entre ces variables et indique une minéralisation des eaux. 


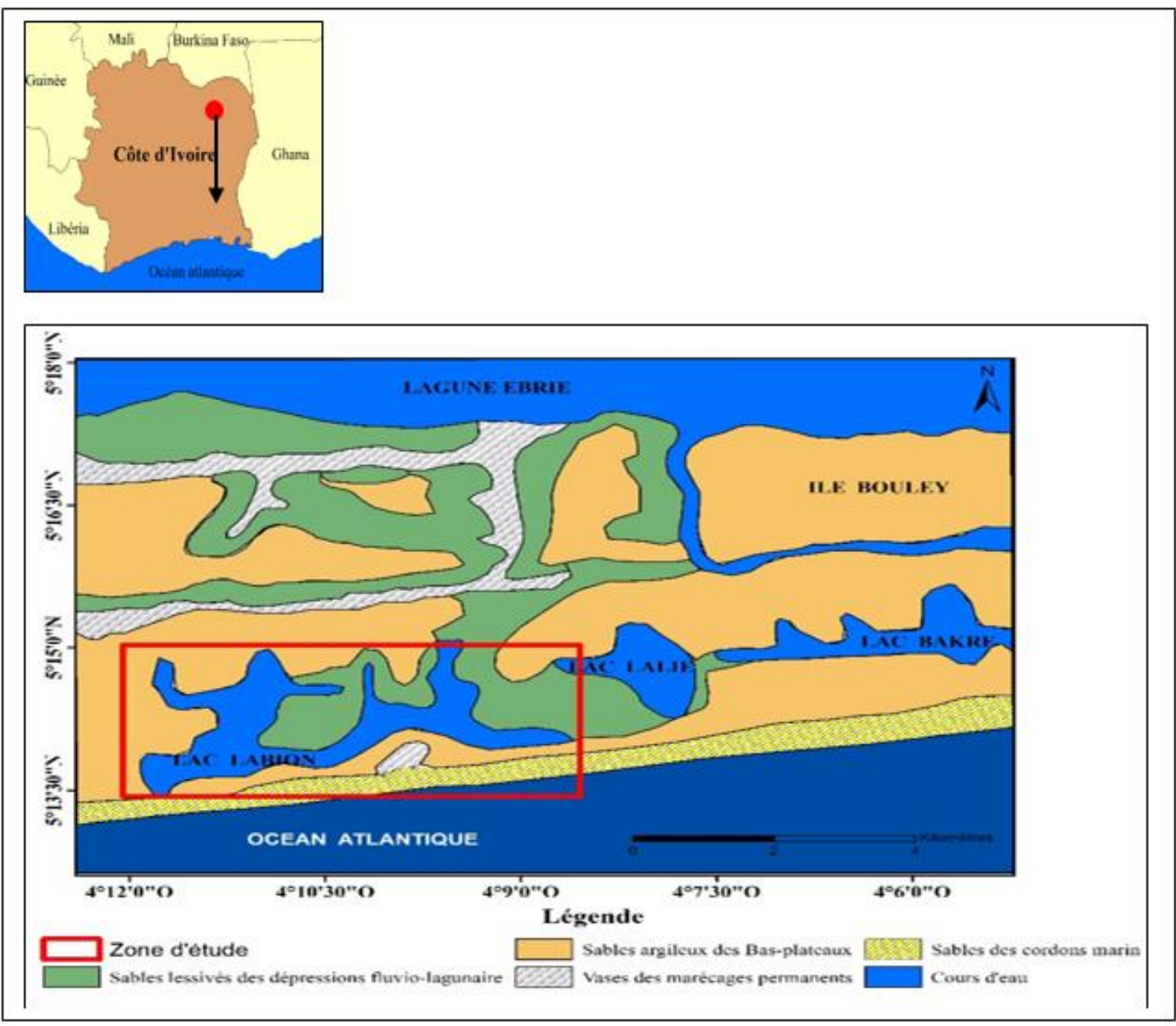

Figure 1 : Localisation du lac Labion.

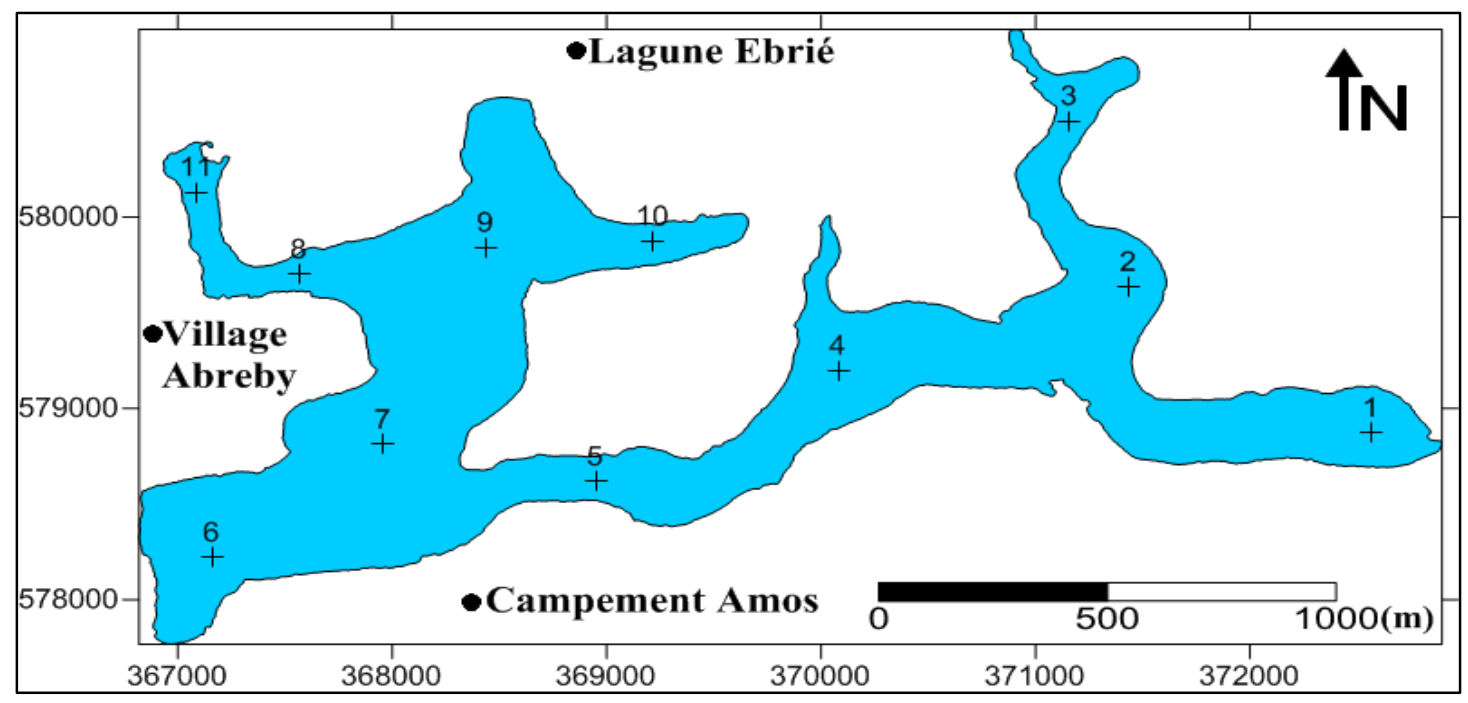

Figure 2 : Station d'échantillonnage. 


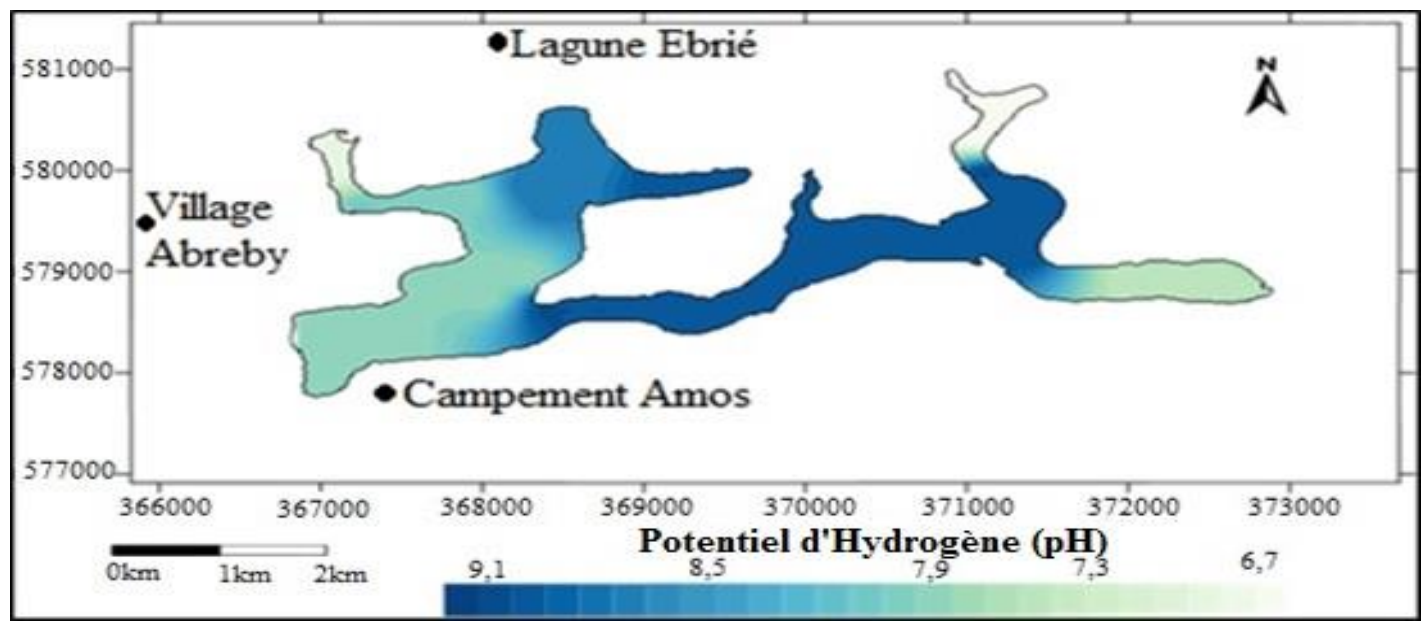

Figure 3 : Répartition spatiale du pH au cours de la grande saison sèche.

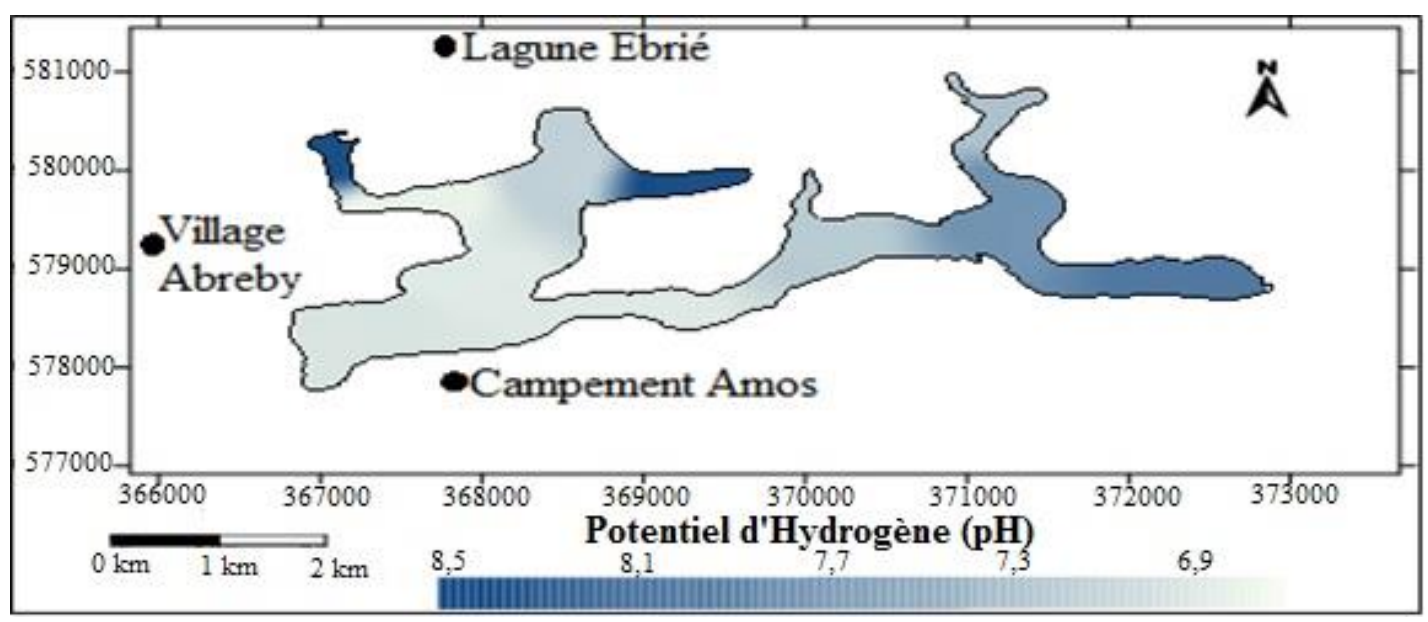

Figure 4 : Evolution du pH pendant la petite saison sèche.

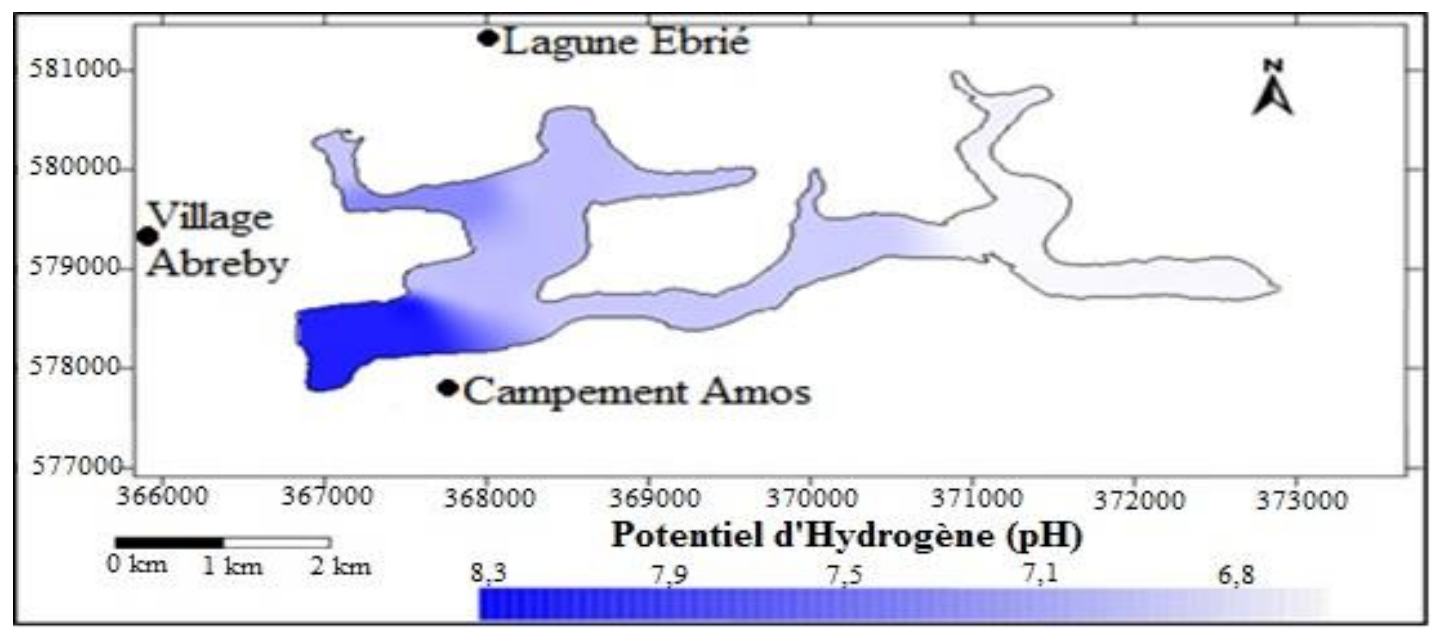

Figure 5 : Evolution spatiale du pH au cours de la grande saison pluvieuse. 


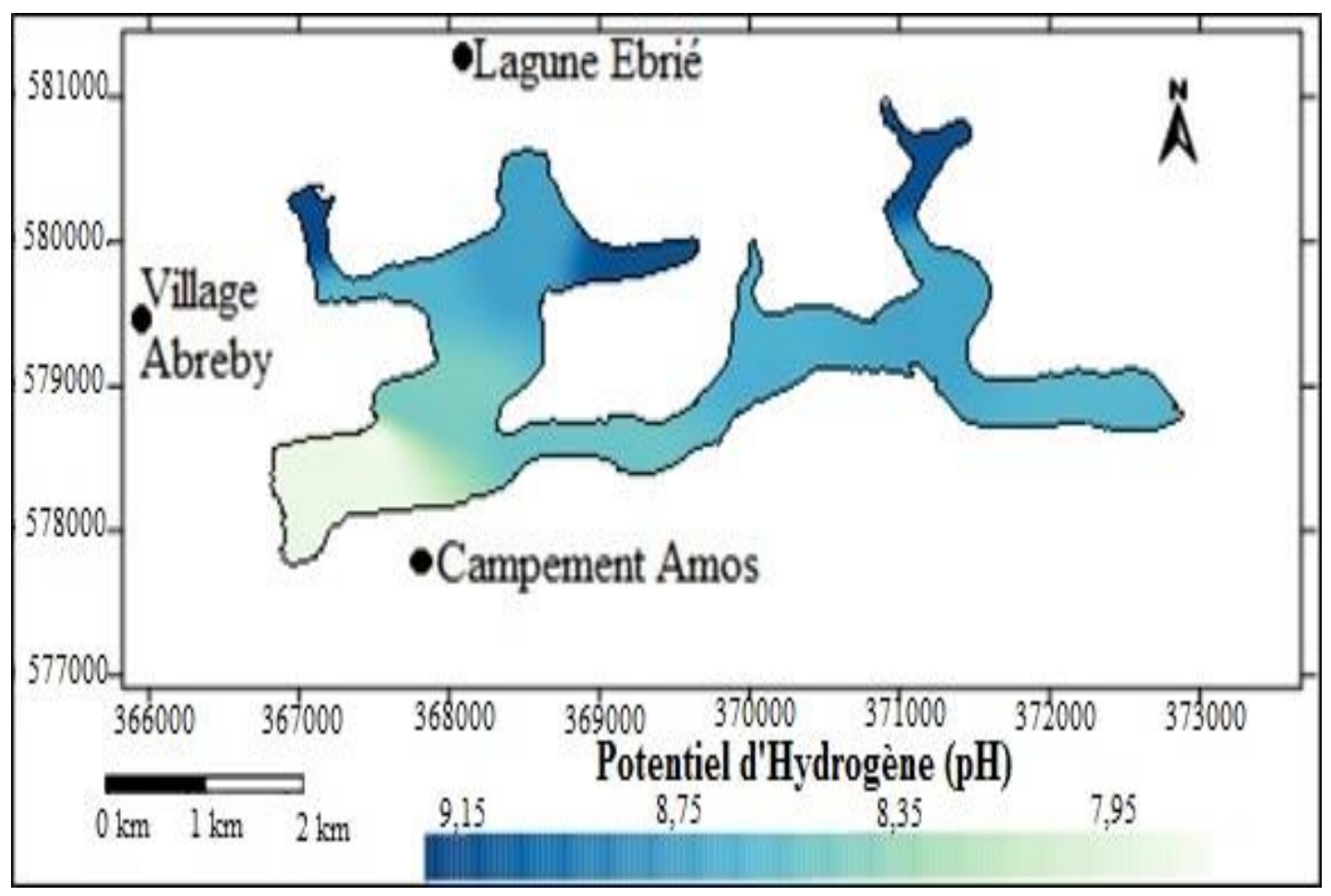

Figure 6 : Répartition spatiale du pH pendant la petite saison pluvieuse.

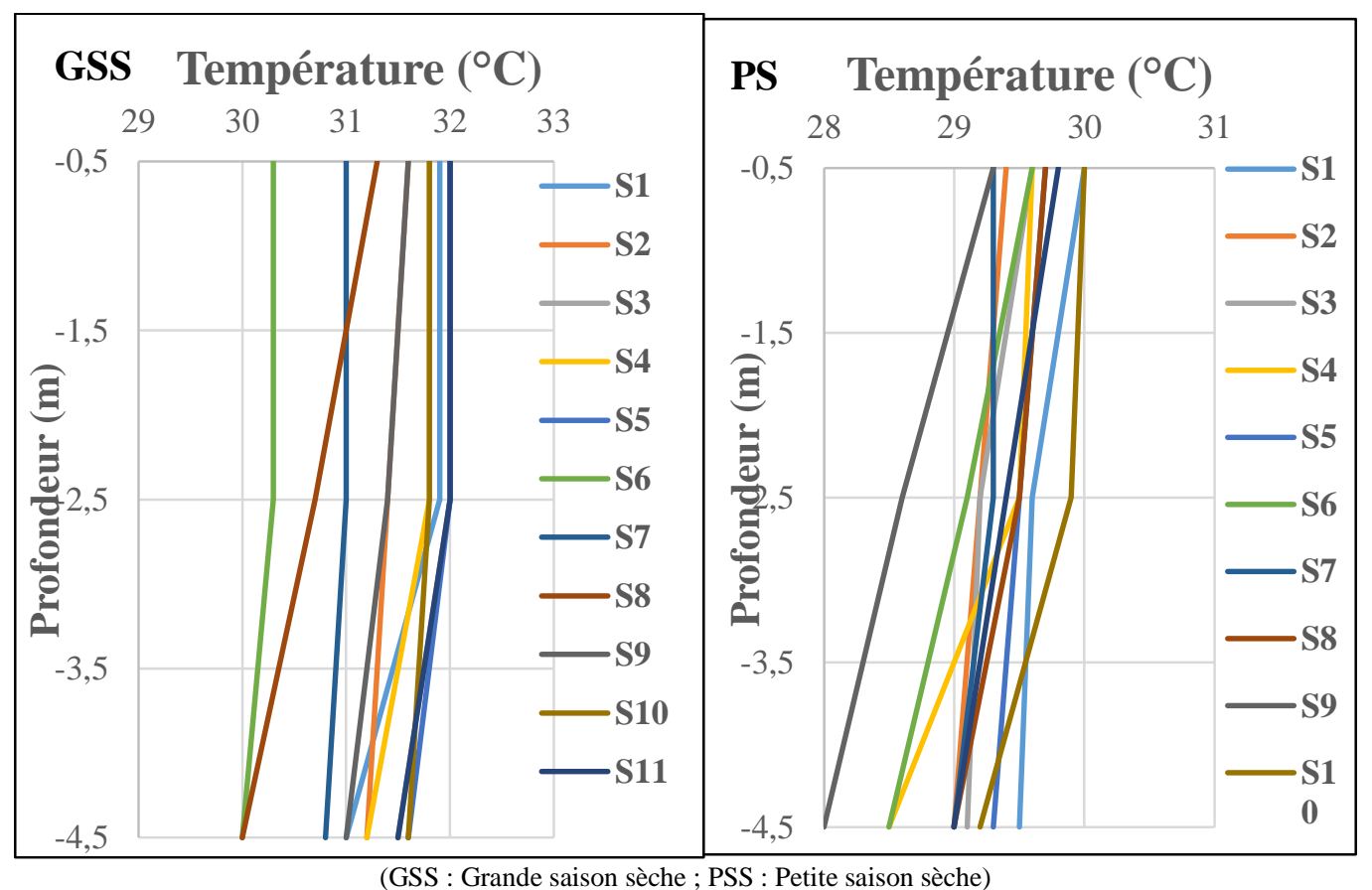

Figure 7 : Evolution de la température dans la colonne d'eau pendant la saison sèche. 


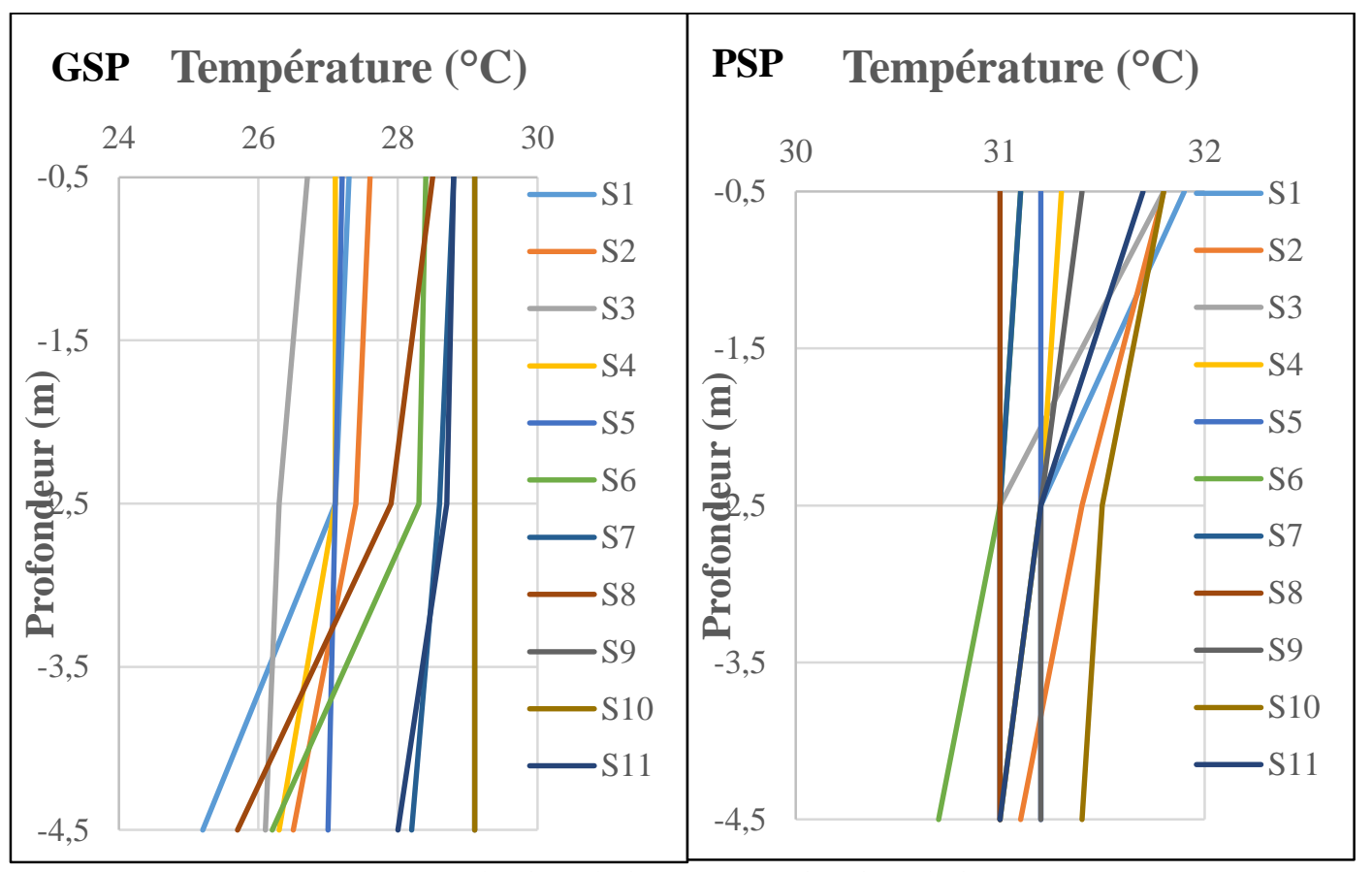

(GSP : Grande saison pluvieuse ; PSP : Petite saison pluvieuse)

Figure 8 : Variation de la température dans la colonne d'eau.

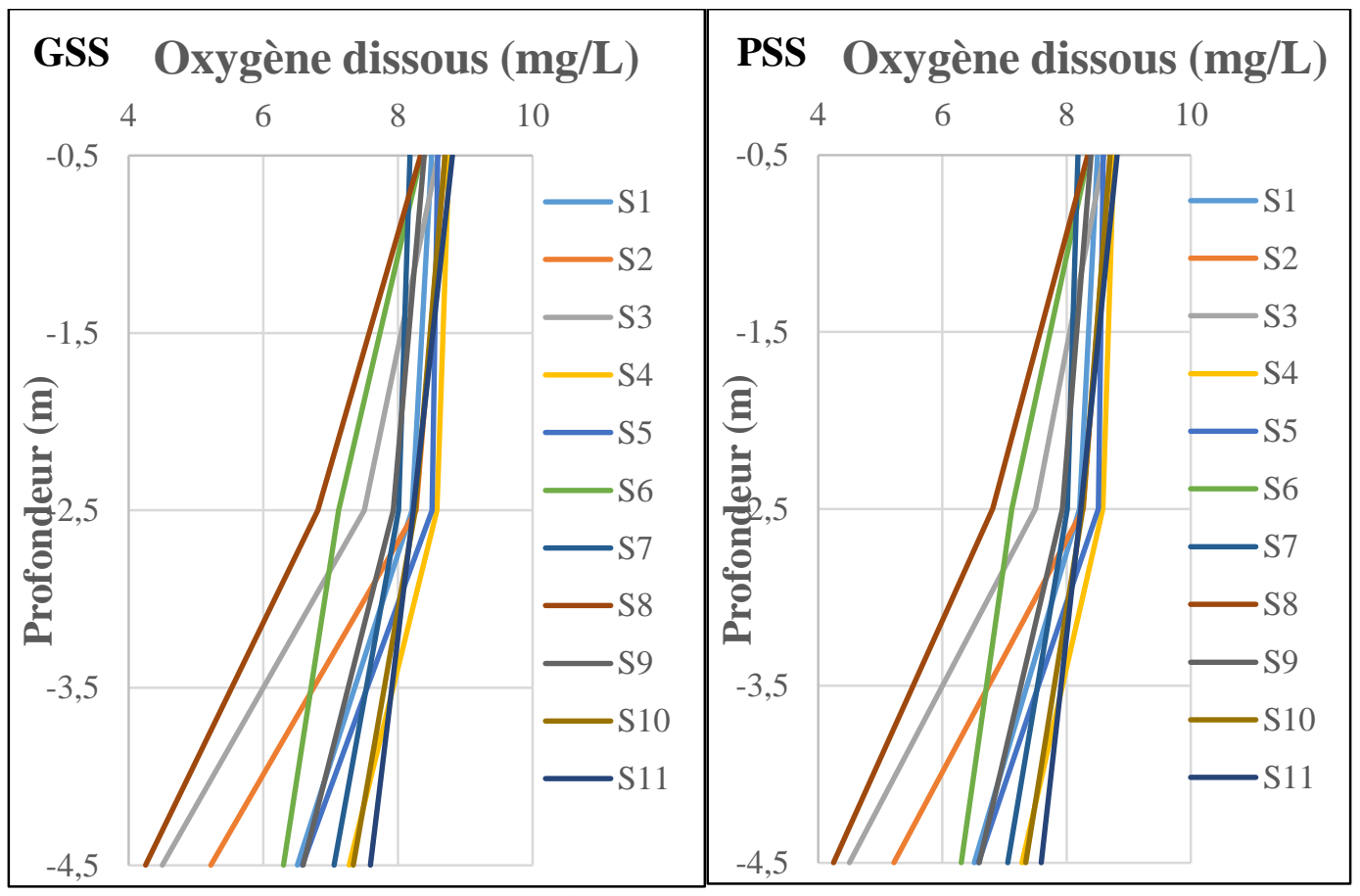

(GSS : Grande saison sèche ; PSS : Petite saison sèche)

Figure 9 : Evolution de l'oxygène dissous dans la colonne d'eau. 


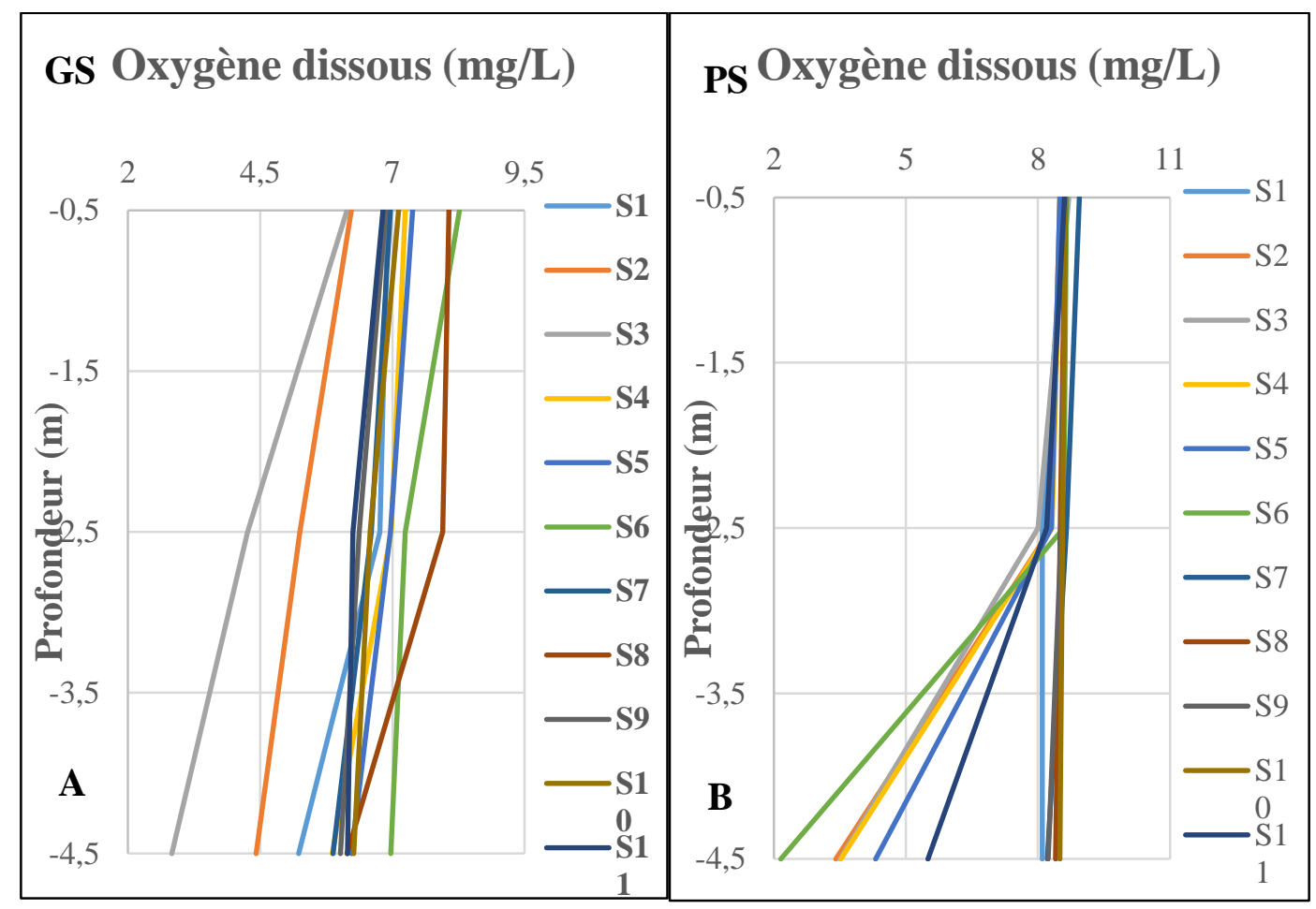

(GSP : Grande saison pluvieuse ; PSP : Petite saison pluvieuse)

Figure 10 : Evolution de l'oxygène dissous dans la colonne d'eau.

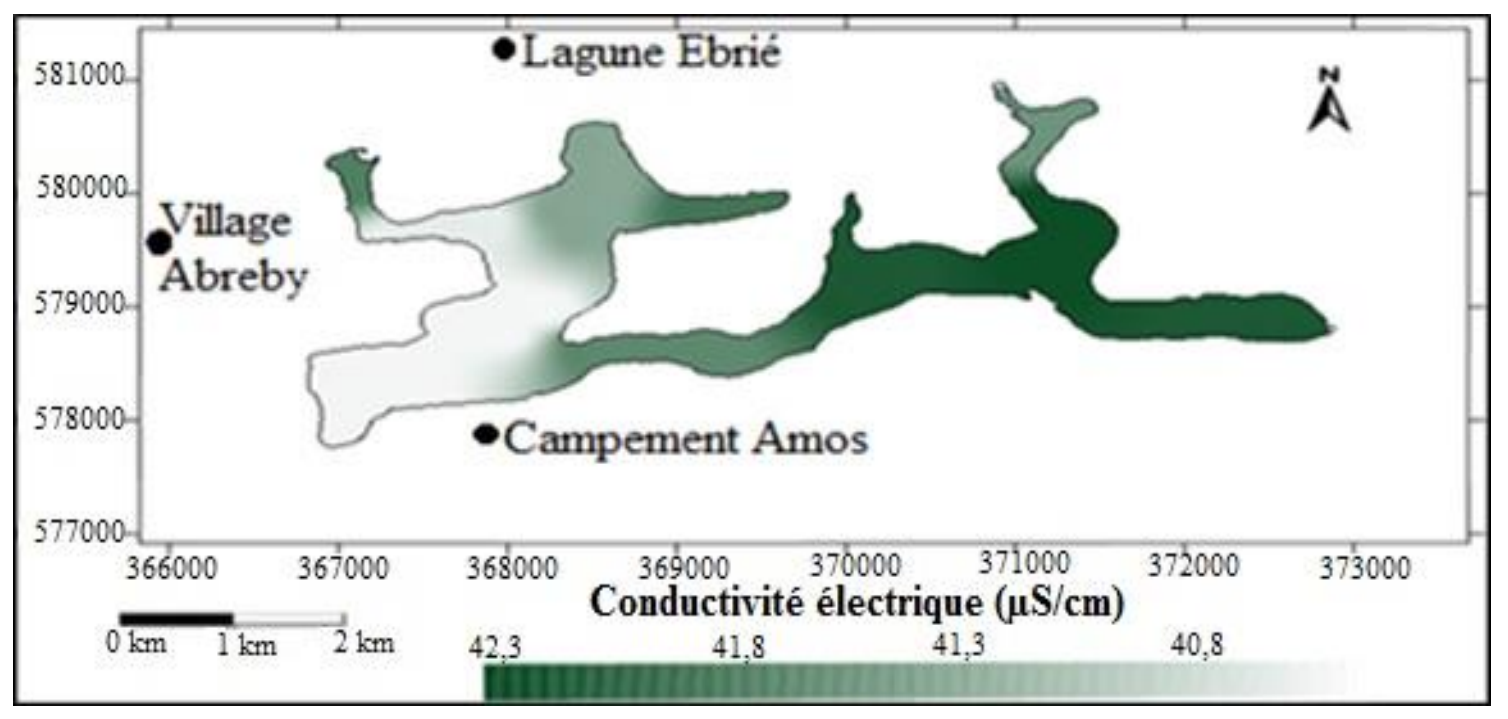

Figure 11 : Répartition spatiale de la conductivité au cours de la grande saison sèche. 


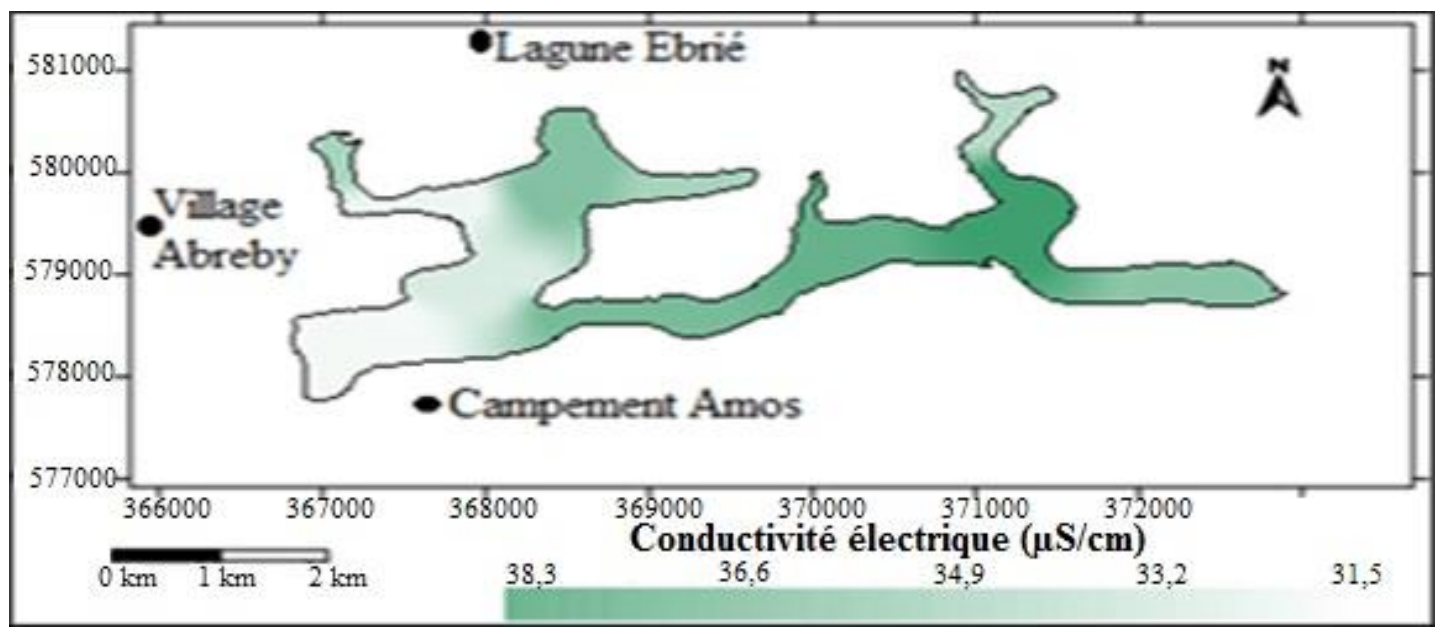

Figure 12 : Répartition spatiale de la Conductivité pendant la petite saison sèche.

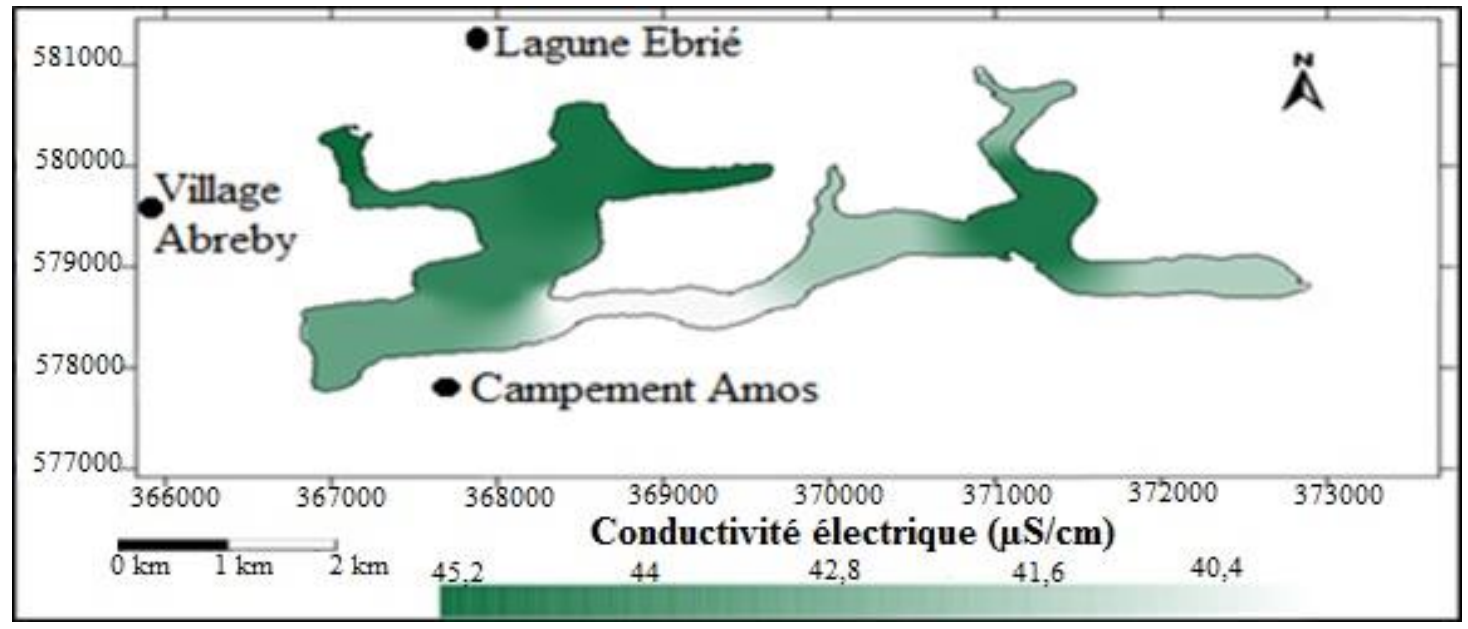

Figure 13 : Evolution spatiale de la conductivité au cours de la grande saison pluvieuse.

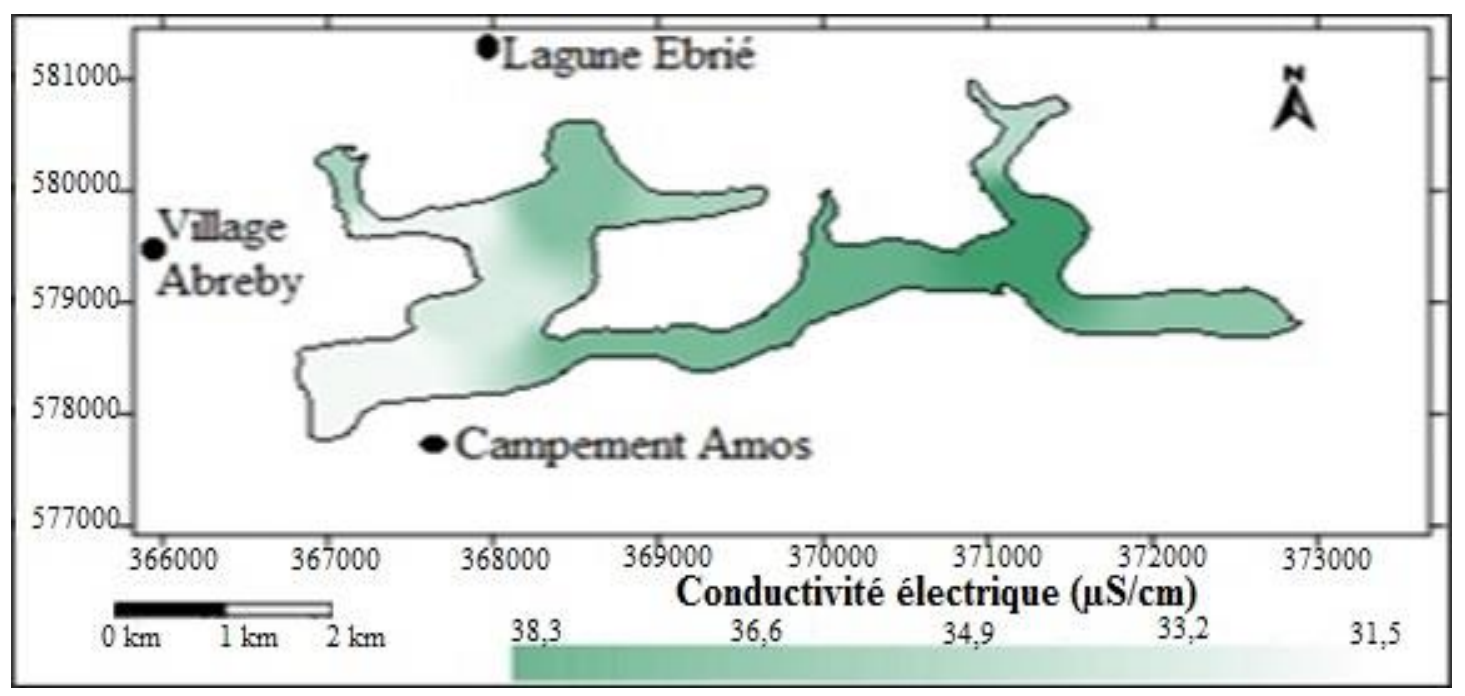

Figure 14 : Variation spatiale de la conductivité électrique pendant la petite saison pluvieuse. 


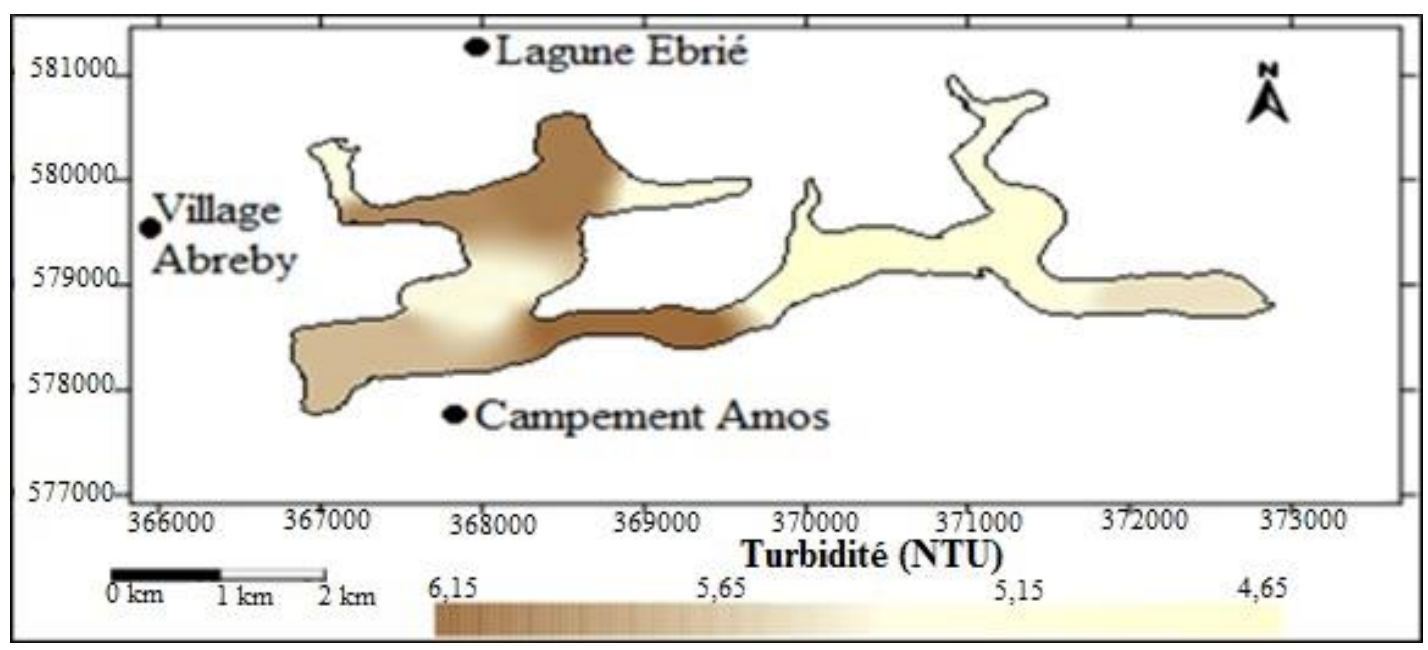

Figure 15 : Répartition spatiale de la Turbidité au cours de la grande saison sèche.

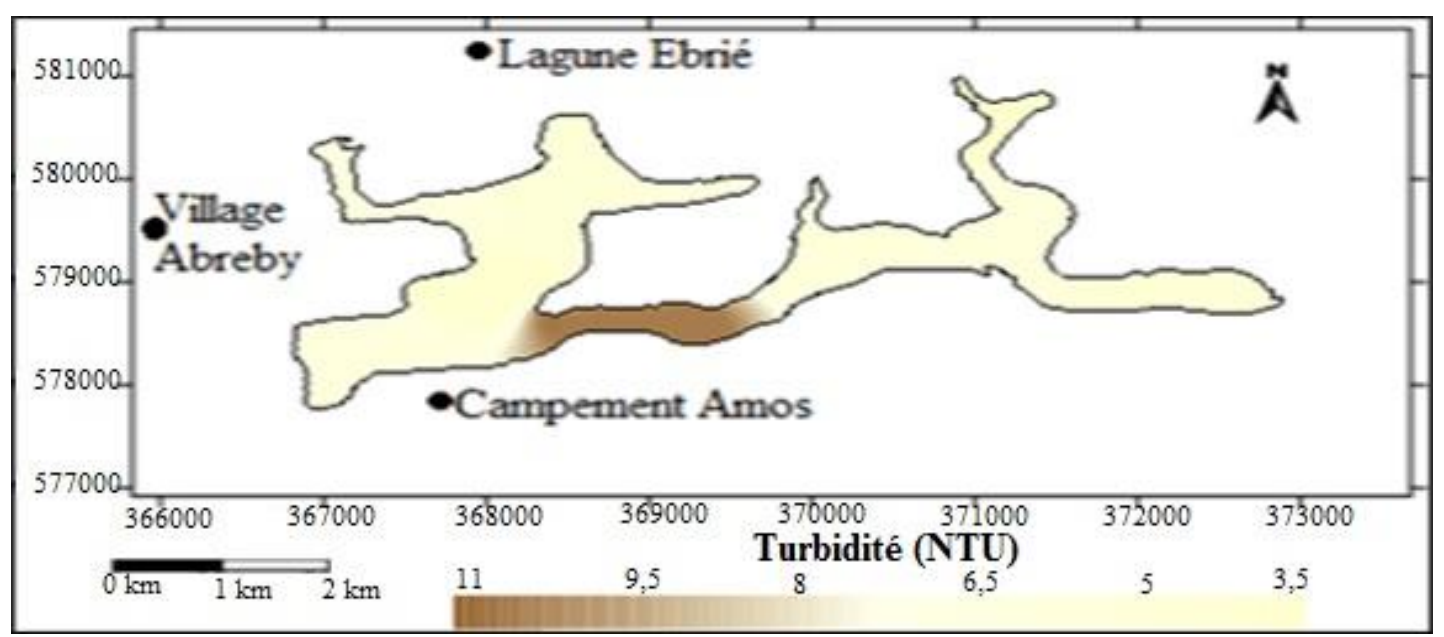

Figure 16 : Evolution spatiale de la Turbidité au cours de la petite saison sèche.

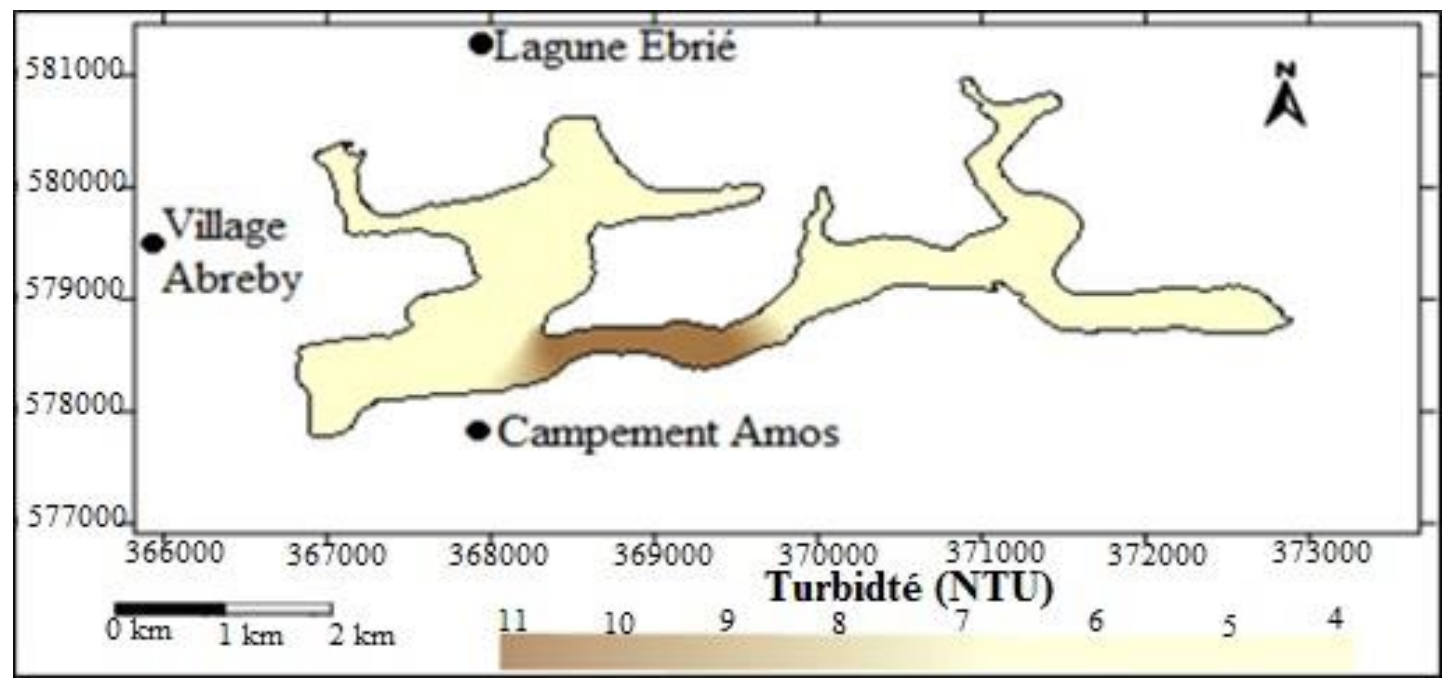

Figure 17 : Variation spatiale de la Turbidité pendant la grande saison pluvieuse. 


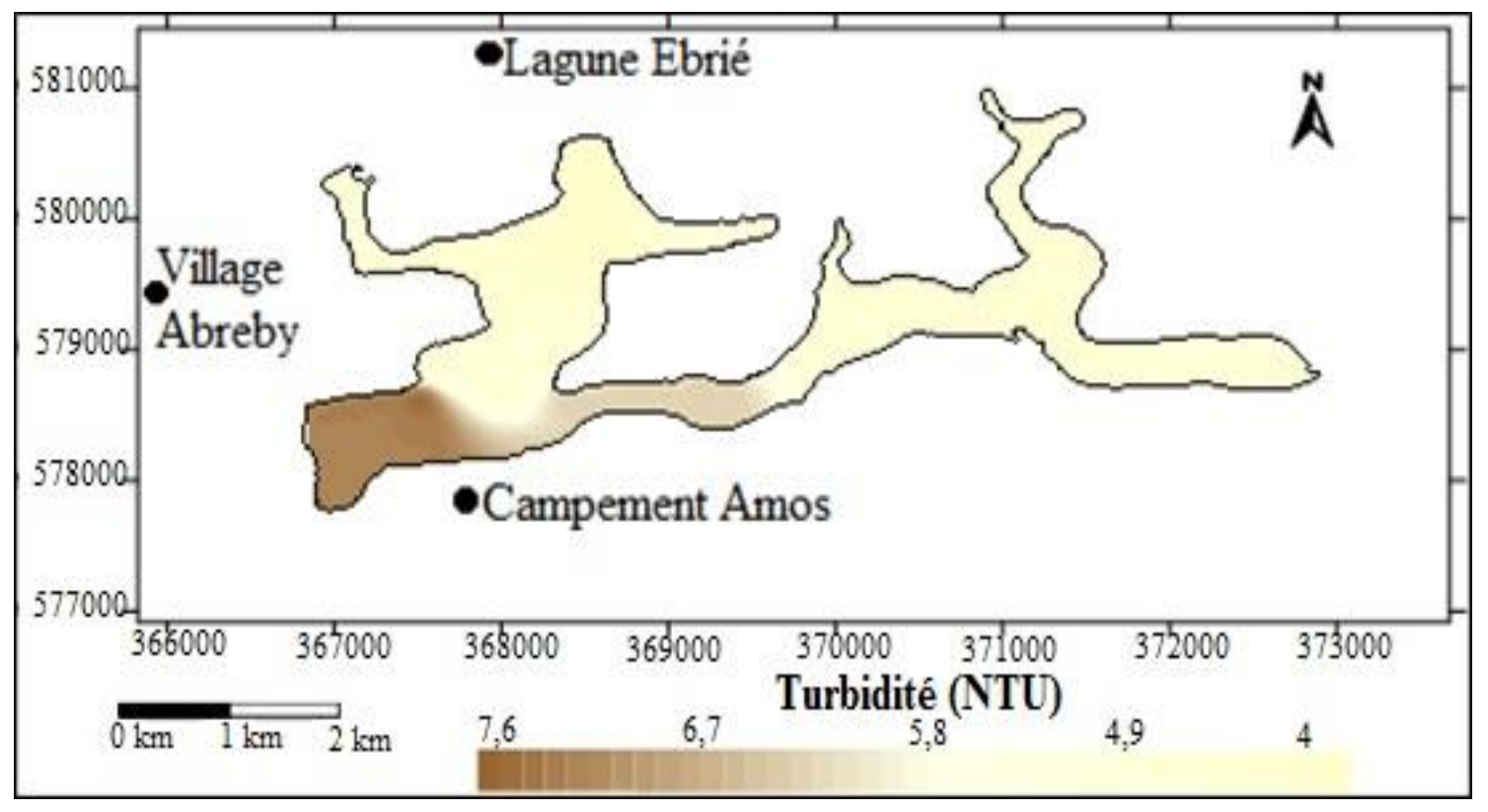

Figure 18 : Répartition spatiale de la Turbidité pendant la petite saison pluvieuse.

Tableau 1: Description des paramètres physico-chimiques.

\begin{tabular}{lcccc}
\hline Paramètres & Moyenne & Minimum & Maximum & Ecart-type \\
\hline pH & 8,22 & 6,79 & 8,99 & 0,45 \\
Température & 31,53 & 30,3 & 32 & 0,98 \\
Oxygène dissous & 8,51 & 8,18 & 8,76 & 0,41 \\
Conductivité & 41,42 & 40,5 & 42,2 & 0,64 \\
Transparence & 0,93 & 0,5 & 1,05 & 0,26 \\
Turbidité & 5,47 & 4,72 & 6,12 & 0,37 \\
\hline
\end{tabular}

Tableau 2 : Matrice de corrélation entre les variables.

\begin{tabular}{lcccccc}
\hline & $\mathbf{p H}$ & Température & Oxygène & Conductivité & Transparence & Turbidité \\
\hline $\mathrm{pH}$ & 1,00 & & & & & \\
Température & 0,04 & 1,00 & & & & \\
Oxygène & 0,16 & 0,69 & 1,00 & & & \\
Conductivité & 0,29 & $\mathbf{0 , 7 7}$ & $\mathbf{0 , 8 3}$ & 1,00 & -000 & \\
Transparence & $\mathbf{0 , 7 0}$ & $-0,33$ &,- 41 & $-0,12$ & $-0,09$ & 1,00 \\
Turbidité & $-0,27$ & $-0,06$ & $-0,33$ & $-0,45$ & & \\
\hline
\end{tabular}




\section{DISCUSSION}

L'analyse du potentiel d'hydrogène en surface a montré l'influence de la photosynthèse sur l'environnement lacustre (Aka, 2016). Elle engendre des $\mathrm{pH}$ neutres parfois basiques sur le lac Labion. En effet, le pH varie de 6,79 à 8,99 pendant la saison sèche et de 6,5 à 8,09 au cours de la saison pluvieuse. Ces valeurs se situent dans l'intervalle des valeurs guides $(6,5-8,5)$ de l'OMS (2004). Les valeurs de $\mathrm{pH}$ obtenues sur le lac Labion sont quasiment proches à celles mesurées sur le lac d'Ayamé I qui varie de 7,1 à 7,8 (Konan et al., 2013) et semblables à celles mesurées sur le lac Bakré (Sud de la Côte d'Ivoire) variant de 6,6 à 8,8 (Aka, 2016). Ces différentes valeurs sont liées aux réactions biologiques et physicochimiques en rapport avec la présence des végétaux aquatiques (Lynda et al., 2011). Le pH du lac Labion est dans les normes de potabilité des eaux de surface.

Concernant la température sur le plan d'eau du lac, elle varie de $30,3{ }^{\circ} \mathrm{C}$ à $32{ }^{\circ} \mathrm{C}$ en saison sèche et de $26,7^{\circ} \mathrm{C}$ à $29,1^{\circ} \mathrm{C}$ en saison pluvieuse. Les températures du lac Labion varient peu et sont sensiblement identiques à celles obtenus $\left(27,48{ }^{\circ} \mathrm{C}\right.$ à $\left.32,45^{\circ} \mathrm{C}\right)$ par Aka (2016). La température de l'eau étant un facteur très important pour le fonctionnement des écosystèmes, est soumise aux influences atmosphériques et particulièrement aux changements de la température de l'air. Ainsi, les fortes températures de l'eau (> $30{ }^{\circ} \mathrm{C}$ ), s'expliqueraient par l'ensoleillement des couches superficielles durant la saison sèche. Les rayons traversent la faible épaisseur d'eau en la réchauffant de façon homogène. En revanche, les températures inférieures à $30^{\circ} \mathrm{C}$ sur le lac Labion obtenues pendant la saison pluvieuse, s'expliqueraient par le refroidissement des eaux dues à l'arrivée des eaux pluviales.

Les concentrations en oxygène dissous des eaux du lac Labion sont en moyenne supérieures à $8 \mathrm{mg} / \mathrm{L}$ durant la saison sèche et à $7 \mathrm{mg} / \mathrm{L}$ au cours de la saison pluvieuse.
L'origine de l'oxygène dissous dans les milieux naturels serait certes liée à l'activité photosynthétique des végétaux aquatiques mais aussi à la dissolution à partir de l'oxygène atmosphérique. Selon l'OMS (2004), une eau de bonne qualité devrait avoir une concentration en oxygène supérieure à $7 \mathrm{mg} / \mathrm{L}$. Sur le lac Labion, les concentrations en OD varient de $8,2 \mathrm{mg} / \mathrm{L}$ à $9,01 \mathrm{mg} / \mathrm{L}$. Ces valeurs sont largement supérieures aux valeurs guides fixées par l'OMS (2004) pour la potabilité des eaux de surface. Par conséquent, les eaux du lac Labion sont donc de bonne qualité.

Le lac Labion présente une conductivité électrique très faible (CE inférieure à 100 $\mu \mathrm{S} / \mathrm{cm}$ ). Elle varie en moyenne de $41,42 \mu \mathrm{S} / \mathrm{cm}$ à $43,12 \mu \mathrm{S} / \mathrm{cm}$ en surface. Sur les lacs de Taabo et Bakré, la conductivité est également faible (83 $\mu \mathrm{S} / \mathrm{cm}$ et $64 \mu \mathrm{S} / \mathrm{cm}$ respectivement). La nature du substratum étant fonction de la minéralisation d'une eau, la conductivité électrique reflète donc des caractéristiques géologiques du bassin versant (Clement et al., 2004 ; CBRSC, 2010). Ainsi, les faibles conductivités enregistrées sur les eaux des lacs au Sud de la Côte d'Ivoire, pourraient s'expliquer par le flux des apports dus au ruissellement d'une part et d'autre part par la nature du substratum qui est constitué de roche sablo-argileuse.

La turbidité et la transparence sont des marqueurs de particules dissoutes dans l'eau. Pendant la saison sèche, la turbidité est peu élevée avec des valeurs de 3,73 NTU à 10,5 NTU. Quant à la transparence au cours de cette même saison, elle varie de $0,5 \mathrm{~m}$ à $1,02 \mathrm{~m}$ de profondeur. On constate donc une turbidité élevée pour une transparence faible. En saison pluvieuse, on observe une turbidité faible qui oscille de 4 NTU à 7,29 NTU et une transparence élevée qui varie de $0,5 \mathrm{~m}$ à $1,2 \mathrm{~m}$. Ces résultats corroborent celle de Aka (2016) sur le lac M'bakré (Sud de la Côte d'Ivoire). Ces différentes valeurs permettent de conclure que le lac Labion est peu chargé de particules 
dissoutes et de matières organiques au cours des saisons.

L'analyse en composantes principales (ACP) réalisée sur le lac Labion au cours des saisons, a permis de ressortir les corrélations existantes entre les variables prises deux à deux. Cependant, les corrélations observées se situent entre les variables oxygène dissous et conductivité électrique. Ces variables ont une origine commune car elles sont corrélées positivement $(r=0,87)$. Les couples de variables température - conductivité et potentiel d'hydrogène - température, présentent également une évolution commune.

Leur coefficient de corrélation est respectivement de 0,77 ) et 0,70 . Le mécanisme mis en évidence est la pollution organique des eaux qui serait due à la présence des matières en suspension (faible transparence) sur le lac (Soro et al., 2010).

\section{Conclusion}

L'étude réalisée, portant sur la caractérisation physico-chimique d'un système lacustre du cordon littoral ivoirien, avait pour objectif principal de caractériser la physicochimie des eaux du lac Labion. Cette étude a permis de ressortir les caractéristiques des eaux du lac pendant les saisons sèches et pluvieuses. L'analyse physico-chimique a montré que la température $\left(>27^{\circ} \mathrm{C}\right)$ et l'oxygène dissous $(>7$ $\mathrm{mg} / \mathrm{L}$ ) présente des valeurs élevées. La transparence et la turbidité évoluent suivant les mêmes séquences de variation aboutissant à une pollution organique. Par ailleurs, la conductivité électrique est le facteur dominant de la qualité douce des eaux du lac. Le pH des eaux du lac sont relativement neutres à basique $(\mathrm{pH}: 6,96$ à 8,22) en surface et acide $(<7)$ en profondeur. Les paramètres conductivité électrique, température et oxygène dissous ont une origine commune car ils présentent une bonne corrélation $(>0,70)$. Les eaux du lac Labion sont relativement chaudes, riche en oxygène, très douces et présente un $\mathrm{pH}$ neutre à basique. L'hydrologie du lac Labion est contrôlée par les influences anthropiques. Il est donc indéniable de réduire l'apport des éléments nutritifs dans le lac. Toutefois, ces eaux pourraient servir comme ressource d'eau douce pour l'alimentation en eau potable. Pour un meilleur suivi des systèmes lacustres qui sont des potentiels sources d'approvisionnement en eau potable, des études sur la bathymétrie et la microbiologie doivent être réalisées.

\section{CONFLIT D'INTERETS}

Les auteurs déclarent qu'il n'y a aucun conflit d'intérêts pour cet article.

\section{CONTRIBUTION DES AUTEURS}

Les auteurs de cet article ont tous contribué aux différents travaux et à la rédaction du manuscrit.

\section{REMERCIEMENTS}

Nous adressons nos sincères remerciement au Laboratoire de Géologie Marine et de Sédimentologie de l'UFR (Unité de Formation et de Recherche) STRM (Sciences de la Terre et des Ressources Minières) de l'Université Félix HouphouëtBoigny de Cocody Abidjan et au Centre Ivoirien AntiPollution (CIAPOL).

\section{REFERENCES}

Abé J. 2005. Contribution à la connaissance de la morphologie et de la dynamique sédimentaire du littoral ivoirien (littoral d'Abidjan). Essais de modélisation en vue d'une gestion rationnelle. Thèse de Doctorat. Université de Cocody. 336p.

Aka C. 2016. Caractérisation bathymétrique, hydrologique et sédimentologique d'un environnement lacustre du littoral de Cote d'Ivoire : cas du lac M'bakré. Thèse de doctorat. Université Felix Houphouet Boigny de Cocody, 176p.

CBRSC. 2010. Conseil du bassin de la rivière de Saint-Charles. Diagnose écologique du lac Neigette, 55p. 
Clément V, Ouimet G. 2004. Programme d'évaluation et de surveillance des lacs. Rapport présenté par Biofilia inc. pour la ville de Mont-tremblant, dossier 2004$1144,26 \mathrm{p}$.

Durand J, Guiral D. 1994. Hydroclimat et Hydrochimie. In Environnement et Ressources Aquatiques de Côte d'Ivoire. Tome II : les Milieux Lagunaires, Durand JR, Dufour P, Guiral D, Zabi SGF (eds). ORSTOM : 59-90.

El Ghachtoul Y, Aloui M, Gabi H. 2008. Eutrophisation des eaux des retenues de barrages Smir et Sehlas Maroc : Causes, conséquences et consignes de gestion. Rev. Sci. de l'Eau, 18 : 75-89.

Kazit T, Arain M, Jamali M, Jalbani N, Afridi H, Sarfraz R, Baig J, Shaha A. 2009. Assessment of water quality of polluted lake using multivariate statistical techniques: a case study. Ecotoxicology and Environnemental Safety, 72: 301309.

Konan K, Kouassi K, Konan K, Kouamé K, Aka K, Gnakri D. 2013. Evaluation des charges solides et caractérisation hydrochimiques des eaux du lac du barrage hydroélectrique d'Ayamé 1 (Côte d'Ivoire). Bulletin de l'Institut Scientifique, Rabat, Section Sciences de la Terre, 35 : 17-25.

Lynda E, Tchirioua E, Jules N, Toussaint D. 2011. Variations temporelles des paramètres physicochimiques et biotiques de deux écosystèmes aquatiques de la lagune Ebrié. European Journal of Scientific Research, 58 (3) : 414-422.

OMS (Organisation Mondiale de la Santé). 2004. Directives de qualité pour l'eau de boisson, Vol. 1-recommandations. Organisation Mondiale de la Santé, $3^{\text {ème }}$ édition, $110 \mathrm{p}$.

Soro G. 2003. Evaluation des métaux lourds (Co, Cr, Mn, Ni et Zn) dans les sédiments de la lagune Ebrié, Abidjan (Côte d'Ivoire). Mém. DEA, Univ. Cocody, 70 p.

Touchart L. 2000. Qu'est-ce qu'un lac ? Bulletin de l'Association de Géographes Français, CNRS, $4: 320$ p. 